\title{
Application of normal-phase high-performance liquid chromatography followed by gas chromatography for analytics of diesel fuel additives
}

\author{
Grzegorz Boczkaj • Mariusz Jaszczołt • \\ Andrzej Przyjazny • Marian Kamiński
}

Received: 5 March 2013 /Revised: 16 April 2013 / Accepted: 30 April 2013 /Published online: 21 May 2013

(C) The Author(s) 2013. This article is published with open access at Springerlink.com

\begin{abstract}
The paper presents the results of investigations on new procedures of determination of selected cleaning additives in diesel fuel. Two procedures: one-step analysis using gas chromatography with flame ionization detection (GCFID) or mass spectrometry (GC-MS) and a two-step procedure in which normal-phase high-performance liquid chromatography (NP-HPLC) was used for preliminary separation of the additives, were compared. The additive fraction was collected using either simple elution or eluent backflush. Final determinations were performed by GC-FID and GC-MS. The studies revealed that it was impossible to determine the investigated analytes by one-step procedures, i.e. by using solely HPLC or GC. On the other hand, the use of a two-step procedure ensures reproducible results of determinations, and the limits of quantitation are, depending on the method of fraction collection by HPLC, from 1.4$2.2 \mathrm{ppm}$ (GC-MS in SIM mode) to 9.6-24.0 ppm (GC-FID). Precision and accuracy of the developed procedures are compared, and possible determination errors and shortcomings discussed.
\end{abstract}

Keywords Fuels $\cdot$ HPLC $\cdot$ GC $\cdot$ Mass spectrometry $\cdot$ Diesel $\cdot$ Sample preparation $\cdot$ Additives

G. Boczkaj $(\bowtie) \cdot$ M. Jaszczołt $\cdot$ M. Kamiński

Chemical Faculty, Department of Chemical and Process

Engineering, Gdansk University of Technology,

G. Narutowicza St. 11/12,

80 - 233 Gdansk, Poland

e-mail: grzegorz.boczkaj@gmail.com

A. Przyjazny

Kettering University, 1700 University Avenue,

Flint, MI 48504, USA

\section{Introduction}

Among automotive fuel additives, an important role is played by cleaning, anticorrosive, octane or cetane number improving additives [1], and - in case of diesel oil additives - those improving lubricating and low-temperature properties. Depressor additives lowering so-called cold filter plugging point are also used. The additives are introduced as solutions in fuel or in one fraction or component of fuel. The most important concentrate components are, depending on its applicability, amines, esters of nitric acid and aliphatic alcohols, special oils, normal and branched alcohols containing 4 to 14 carbon atoms, including 1-hexanol, 2-ethylhexanol, or isopropanol as well as special block polymers or organometallic compounds, including zinc alkyl dithiophosphates [2-4]. In many cases, alcohols are also used as solvents for active ingredients of the additives, assuring homogeneity of the mixture being the final form of the additive as well as solubility in the fuel. 2Ethylhexanol is used as a solvent in foam suppressants based on polydimethylsiloxane, which are added to diesel oil $[5,6]$. The content of active ingredients of individual additives in fuel is low, typically ranging from a few parts per million to a few hundred parts per million $(w / w)$, which greatly complicates analytics.

In order to control blending process or for quality control of the final product, it is sometimes necessary to determine the content of selected additives in fuel. A very complex matrix such as gasoline, and particularly diesel fuel, containing a large number of groups of organic compounds, presents a great analytical challenge for the determination of additives content in fuel.

To this end, it is necessary either to isolate a particular component(s) by adsorption, e.g., by solid-phase extraction or liquid-liquid extraction, or by using adsorption chromatography, preferably normal-phase or reverse-phase high- 
performance liquid chromatography (NP-HPLC or RPHPLC), followed by gas chromatography (GC) $[7,8]$ or liquid chromatography in order to perform final determination of an analyte or group of analytes.

In the case of determination of lubricating additives in diesel fuel, which was a mixture of oleic and linoleic acids, an extraction-derivatization method with the phase transfer catalyst (methanolic solution of tetramethylammonium hydroxide) was used. The resulting extract was introduced directly into the GC injection port. Lubricating additives in the extract are present as tetramethylammonium salts of fatty acids, which decompose at a high temperature in the injection port to yield methyl esters of fatty acids. Flame ionization detector was used for the determination and recoveries varied from 95 to $98 \%$ [9]. A promising technique for the determination of analytes in complex matrices is solid-phase microextraction. When used for a direct extraction from a matrix, it is a solvent-free technique, which allows direct GC analysis with thermal desorption in the GC injection port [10]. In some cases of determination of additives in diesel fuel, an additional step of fraction isolation was not necessary due to application of highly selective methods of final determination. Such an approach was employed, for example, in the determination of a lubricating additive, 2-hydroxyethyl linoleate, in diesel fuel by the internal standard method, using pentadecanoyl propanoate as the internal standard. GC-mass spectrometric detection selected ion monitoring (GC-MS-SIM) was chosen for the final determination. The analysis of MS spectra of the analyte and internal standard revealed that the use of single ion monitoring at $255 \mathrm{~m} / \mathrm{z}$ and $263 \mathrm{~m} / \mathrm{z}$, respectively, allows direct analyses without the need for analyte isolation during the sample preparation step. This results from low intensity of these ions in case of analysis of diesel fuel within the range of retention time of the analyte. Nevertheless, for the investigated range of concentrations of the additive: 54$180 \mu \mathrm{g} / \mathrm{mL}$, the recovery values slightly exceeded $100 \%$, and in one case the recovery was equal to $125 \%$ [11]. A similar approach was used for the determination of antioxidants in aviation fuel by two alternative procedures - reversephase high-performance liquid chromatography with electrochemical detection (RP-HPLC-ED) or GC-MS-SIM. Similar to the determination of lubricating additives, the SIM mode allows a selection of the signal of the analytes only due to high intensities of the ions derived from the analytes with large values of the mass-to-charge ratio, and not produced from the matrix components [12]. In addition, procedures for the determination of additives to lubricating oils based on amines and organophosphorus compounds have been developed. GCMS-SIM or, alternatively, gas chromatography with the flame photometric detector (GC-FPD) in the phosphorus mode was used. The determinations were performed directly on lubricating oil dissolved in hexane by the internal standard method.
The investigated amines have very abundant ions with a high mass-to-charge ratio; for example, for dioctyl diphenyl amine they are 393 and $322 \mathrm{~m} / \mathrm{z}$. The paper did not mention any problems related to peak overlap of matrix components and the analyte or elution of high-molecular-mass components (the final distillation temperature of lubricating oil components can exceed $500{ }^{\circ} \mathrm{C}$ ), which can be expected when using operating conditions described in the paper (final GC oven temperature-below $290^{\circ} \mathrm{C}$ ) [13].

This paper compares one- and two-step procedures making use of coupled techniques for the determination of cleaning additives in diesel fuel. The additives contain straight-chain and branched alcohols, solvent naphtha, a mixture of trimethylbenzene isomers and naphthalene. The developed procedure allows the determination of additives containing 2-ethylhexanol in diesel fuel.

One of the important roles of such additives is prevention from the formation of deposits in the injection system, where the temperature and pressure conditions can result the formation of deposits of contaminants coming from the fuel. This is particularly important for the fuels containing diesel fuel additives prone to precipitation during abrupt changes in operating conditions. Even small amounts of deposits in the injector nozzle (nozzle orifice diameters from 80 to $110 \mu \mathrm{m}$ ) result in a significant power loss and, in a long term, to the formation of carbon deposits and a serious failure of the fuel injection system.

\section{Material and methods}

\section{Materials}

The standards used: 2-ethylhexanol, naphthalene, pyrene (Merck, Darmstadt, Germany) had purity over $99 \%$ by weight, and the commercial cleaning additive was of technical purity. The eluents used in the investigations - hexane, and methyl tert-butyl ether (Merck, Darmstadt, Germany) were of HPLC grade. Gases used in GC analyses (hydrogen, air, helium; Linde Gas, Poland) were of $5.0 \mathrm{~N}$ purity.

\section{Apparatus}

A Merck-Hitachi gradient liquid chromatograph (Darmstadt, Germany) equipped with a four-channel lowpressure gradient system, a L-6200 pump, a Rheodyne Rh7161 (Idex, Oak Harbor, USA) injector with a 1-mL sample loop, a chromatographic column, a thermostat, a model L3000 UV diode array detector (DAD) detector, a model 1037A refractive index detector, HSM software, and a V 7226 six-port two-channel backflush valve (Knauer, Berlin, Germany). The following columns were used for the normal-phase separations: one $250 \times 4 \mathrm{~mm}$ Purospher ${ }^{\circledR}$ 
STAR $\mathrm{NH}_{2}(5 \mu \mathrm{m})$ column (Merck, Darmstadt, Germany) and two $250 \times 4 \mathrm{~mm}$ Nucleosil 50-3 columns (MachereyNagel, Duren, Germany).

Collected fractions were analyzed by gas chromatography. An HP 5890 gas chromatograph (Hewlett-Packard, USA) coupled to an HP 5972A mass spectrometer, with a $60.0 \mathrm{~m} \times 0.25 \mathrm{~mm} \times 0.25 \mu \mathrm{m}$ DB5ms capillary column (Agilent, Santa Clara, USA) and an Autosystem gas chromatograph (Perkin Elmer, Waltham, USA) with a flame ionization detector (FID), with a $60.0 \mathrm{~m} \times 0.25 \mathrm{~mm} \times$ $0.25 \mu \mathrm{m}$ DB5ms capillary column (Agilent, Santa Clara, USA), were used in the investigations.

\section{Procedure}

\section{Preparation of standard solutions}

In order to determine retention times of selected additive components, standard mixtures of 2-ethylhexanol and naphthalene (both commonly used in detergent additives to diesel oil) were prepared in original diesel fuel. The concentration of standards was about $100 \mathrm{ppm}(w / w)$. Calibration mixtures for quantitative analysis with concentrations ranging from 0.1 to $1,500 \mathrm{ppm}(\mathrm{w} / \mathrm{w})$ were prepared in the same manner. Concentrations were calculated from the mass of components used to prepare the mixtures.

\section{Single-step procedure-normal-phase high-performance liquid chromatography with refractive index detector and UV-VIS diode array detector}

One, two, or three HPLC columns connected in series were used in the investigations. The possibility of separation of diesel fuel components with the additive by using from one to three NP-HPLC columns and the UV-VIS-DAD detector was studied to a degree allowing direct determination of naphthalene.

Separations were carried out using isocratic elution with the hexane-methyl tert-butyl ether (MTBE) mobile phase, with the MTBE content varying from 0 to $30 \%(v / v)$. After complete elution of the standard, the column was backflushed. Dual detection: UV diode array detector (UV/DAD) and refractive index detector (RID) connected in series, was used in the investigations. UV/DAD detection conditions: spectral range $220-600 \mathrm{~nm}$, monitored UV wavelength (characteristic of naphthalene): $275 \mathrm{~nm}$.

Single-step procedure - capillary gas chromatography with flame ionization detector or mass spectrometric detection

Samples $(1.0 \mu \mathrm{L})$ were injected with a microsyringe. Split $(10: 1)$ injection mode was used. Separations were carried out under the following conditions:
Capillary gas chromatography with flame ionization detector Helium at a flow rate of $1.1 \mathrm{~mL} / \mathrm{min}$ was used as the carrier gas. The initial oven temperature was $70{ }^{\circ} \mathrm{C}$ (held for $5 \mathrm{~min}$ ), followed by a ramp to $185^{\circ} \mathrm{C}$ at $6{ }^{\circ} \mathrm{C} / \mathrm{min}$ (held at $185{ }^{\circ} \mathrm{C}$ for $1 \mathrm{~min}$ ), and then ramped to $330{ }^{\circ} \mathrm{C}$ at $30{ }^{\circ} \mathrm{C} / \mathrm{min}$ (held at $330{ }^{\circ} \mathrm{C}$ for $12 \mathrm{~min}$ ). The injection port and detector temperatures were 350 and $300{ }^{\circ} \mathrm{C}$, respective1y. The hydrogen and air flow rates in FID 40 and $430 \mathrm{~mL} / \mathrm{min}$, respectively.

Capillary gas chromatography and mass spectrometry-SCAN and SIM modes Helium at a flow rate of $1.1 \mathrm{~mL} / \mathrm{min}$ was used as the carrier gas. The initial oven temperature was $80{ }^{\circ} \mathrm{C}$ (held for $3 \mathrm{~min}$ ), followed by a ramp to $180{ }^{\circ} \mathrm{C}$ at $10{ }^{\circ} \mathrm{C} / \mathrm{min}$, and then ramped to $300{ }^{\circ} \mathrm{C}$ at $25{ }^{\circ} \mathrm{C} / \mathrm{min}$ (held at $300{ }^{\circ} \mathrm{C}$ for $15 \mathrm{~min}$ ). The injection port and GCMS transfer line temperatures were 350 and $310{ }^{\circ} \mathrm{C}$, respectively. The temperature of the ion source (EI, $70 \mathrm{eV}$ ) was $200{ }^{\circ} \mathrm{C}$; SCAN mode over the mass-tocharge ratio range of $34-300 \mathrm{~m} / \mathrm{z}$ was used for the determination of retention times.

In the study on the possibility of single-step determination of additive content in diesel fuel by GC-MS in the SIM mode, selected characteristic ions for 2-ethylhexanol (70, 83 $\mathrm{m} / \mathrm{z}$ ) were monitored over $0-13 \mathrm{~min}$ and for naphthalene $(128 \mathrm{~m} / \mathrm{z})$ over $13-30 \mathrm{~min}$.

In quantitative determination of the additive in the fraction collected from NP-HPLC (two-step procedure), only ions characteristic of 2-ethylhexane $(70,83 \mathrm{~m} / \mathrm{z})$ were monitored.

Two-step procedure-fraction isolation using NP-HPLC, followed by GC-FID or GC-MS for final determination The additive fraction isolated by NP-HPLC was collected in a vial, followed by analysis of the eluate using GC-FID or GC-MS.

Isolation of the additive fraction from a sample of diesel fuel using NP-HPLC and backflush elution-NP-HPLC-EBF/ $U V-V I S-D A D / R I D$ One $250 \mathrm{~mm} \times 4 \mathrm{~mm} \times 5 \mu \mathrm{m} \mathrm{NH} \mathrm{N}_{2}$-type column was used. The characteristic fraction of the additive being determined was eluted using backflush, with the backflush time established by the retention time of pyrene, collecting the fraction over the time determined for a standard solution of 2-ethylhexanol. The retention time of pyrene under conditions of group separation in NP-HPLC determines the end point of elution of tricyclic aromatic hydrocarbons. By then all the remaining matrix components of diesel fuel have been eluted from the column. Only resins and polar components of the additive can have longer retention times. Two detectors were used in series: a DAD and a RID. The separation was carried out isocratically with a two-component mobile phase, containing hexane and 
MTBE in volume ratio varying from 20:1 to $4: 1 v / v$. The other separation conditions were as follows: mobile phase flow rate $1.5 \mathrm{~mL} / \mathrm{min}$; injection volume $0.2 \mathrm{~mL}$. The use of two detectors: UV and refractive index, permits an additional detection of the presence of polar components of diesel fuel (resins) in the fraction collected during backflush. The resins are characterized by strong UV absorption in contrast with 2-ethylhexanol being collected.

Isolation of the additive fraction using NP-HPLC/UV-VIS$D A D / R I D$ The additive fraction was eluted over the time determined for a standard mixture of 2-ethylhexanol. Isocratic elution with a binary mobile phase (hexane/ MTBE) was used. The experiments were carried out for various volume ratios of the solvents, with the MTBE content over $12.5 \%$. The column was backflushed following complete elution of the additive fraction collected. The remaining conditions were the same as in the section "Isolation of the additive fraction from a sample of diesel fuel using NP-HPLC and backflush elution-NP-HPLCEBF/UV-VIS-DAD/RID”.

Final determination Both GC-FID and GC-MS determinations were carried out under conditions identical to those described in the section "Single-step procedure - capillary gas chromatography with flame ionization detector or mass spectrometric detection".

\section{Quantitative analysis}

Determinations of the additive content were performed using five-point calibration curves by GC-FID or GC-MS. Calibration was based on peak areas. Each point on the calibration curve was calculated as the average of three results. The concentration of the additive in the column eluate was converted to the mass concentration of the additive in diesel fuel for a $200-\mu \mathrm{L}$ injection volume, taking into account the time of fraction collection and the densities of the additive and diesel fuel, according to the following relationship:

$C_{A D D}\left[\frac{m g}{k g D F}\right]=7,5 \cdot\left(t_{2}-t_{1}\right) \cdot C_{G C} \cdot \frac{\rho_{A D D I T I V E}}{\rho_{D F}}$

where:

$t_{2}, t_{1} \quad$ Time at the end and beginning of HPLC

fraction collection, respectively [in minute]

$C_{\mathrm{GC}} \quad$ Additive concentration in the eluate determined by GC

$\rho_{\text {ADDITIVE }}, \quad$ Densities of the additive and diesel fuel, $\rho_{\mathrm{DF}} \quad$ respectively [in kilogram per cubic meter]

7.5
Coefficient resulting from mass balance for the HPLC system in general $(a / b)$

Where:

a Volumetric flow rate of the mobile phase [in milliliter per minute]: in this case $1.5 \mathrm{~mL} / \mathrm{min}$

$b$ Volume of injected sample [in milliliter] $-0.2 \mathrm{~mL}$.

\section{Validation of two-step procedure}

Two standard mixtures of the additive in diesel fuel were prepared with concentrations of about 50 and $400 \mathrm{ppm}$ $(w / w)$. Three analyses were carried out for each of the mixtures according to the procedure described in the sections "Isolation of the additive fraction from a sample of diesel fuel using NP-HPLC and backflush elution-NPHPLC-EBF/UV-VIS-DAD/RID", "Isolation of the additive fraction using NP-HPLC/UV-VIS-DAD/RID", and "Final determination". The same procedure was used for the blank - the original diesel fuel to which no cleaning additive was added.

\section{Precision}

Relative standard deviation (coefficient of variation) was calculated for the obtained results.

Limits of detection and quantification

Limits of detection (LOD) and quantification (LOQ) were found for the signal to noise ratios: $S / N=3$ for LOD and $S / N=6$ for $\mathrm{LOQ}$.

Accuracy

Accuracy was determined as a relative error (recovery) of the expected value determined from the masses of additive and diesel fuel in a standard mixture.

\section{Results and discussion}

The procedure for determination of cleaning additive in diesel fuel described in this paper is based on quantitative isolation from diesel oil of a characteristic fraction containing, depending on origin, aliphatic alcohols with a various length of carbon chain. In this paper, an additive with 2-ethylhexanol was used. Naphthalene is also a known component of this type of additives, with the content of up to several percent. This compound was also taken as a compound employed for quantitative determinations with 
Fig. 1 NP-HPLC/UV/DAD chromatogram of sample of diesel fuel containing cleaning additive. Conditions as described in the section "Isolation of the additive fraction from a sample of diesel fuel using NP-HPLC and backflush elution-NP-HPLCEBF/UV-VIS-DAD/RID" of the "Procedure" $-5 \%$ MTBE in the mobile phase; $B F$ backflush point

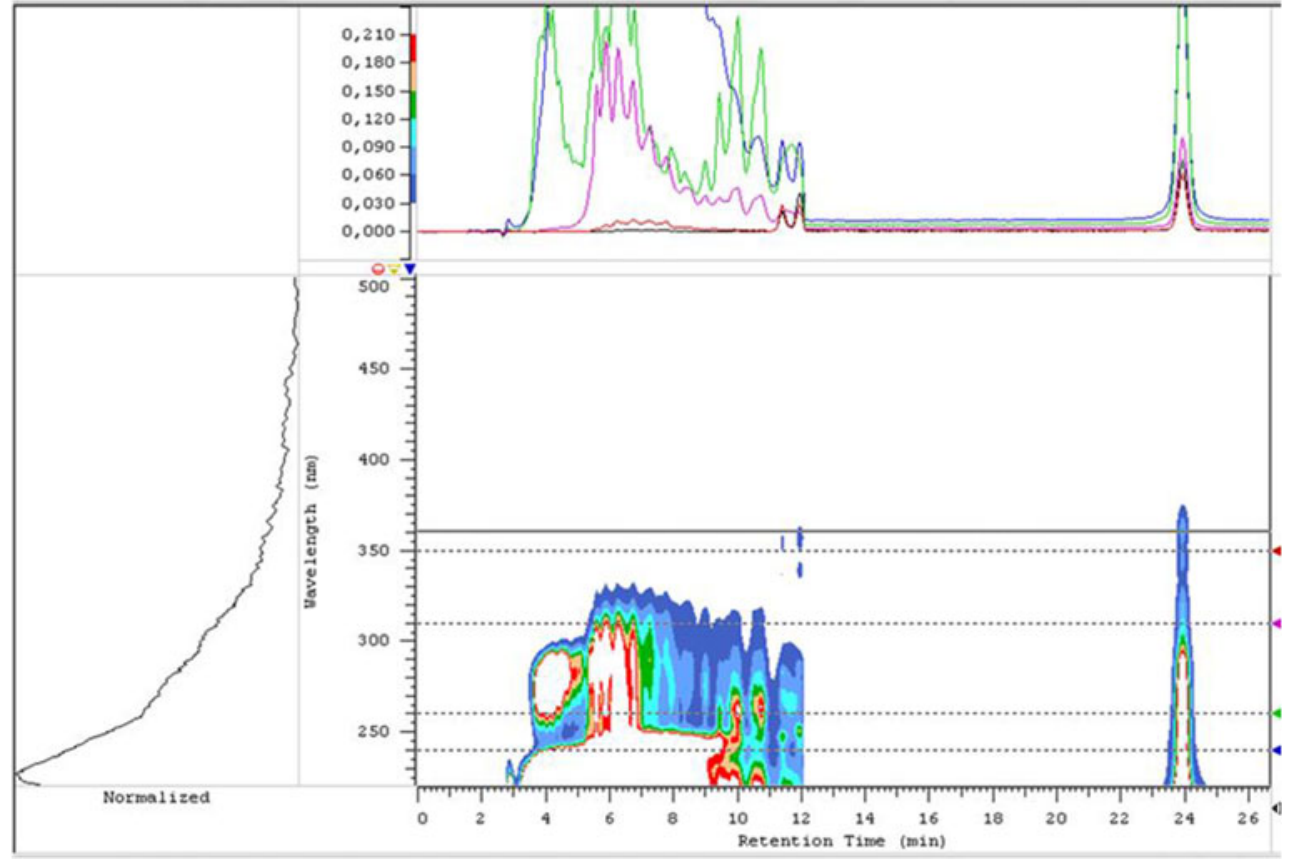

components, a characteristic UV spectrum of naphthalene would enable quantitative determination by a UV detector. However, the results obtained in this study proved such an approach to be unfeasible. The sole use of gas chromatography is impossible due to the elution temperature of main components of the additive overlapping with the elution temperature of diesel fuel. The separation and determination of characteristic peaks of the additive in the presence of diesel fuel is not possible even when using 60.0-m highefficiency capillary columns either with a FID or with mass spectrometry with SIM. Insufficiently, specific fragment ions coming from 2-ethylhexanol result in detection of a large signal from the hydrocarbon matrix of diesel fuel at the retention time of the analyte. Such a problem did not occur
Fig. 2 HPLC-RID

chromatogram of a sample of diesel fuel containing cleaning additive. Conditions as described in "Isolation of the additive fraction from a sample of diesel fuel using NP-HPLC and backflush elution-NPHPLC-EBF/UV-VIS-DAD/ RID" of the "Procedure"$12.5 \%$ MTBE in the mobile phase; $B F$ backflush point, $F C$ fraction collection range

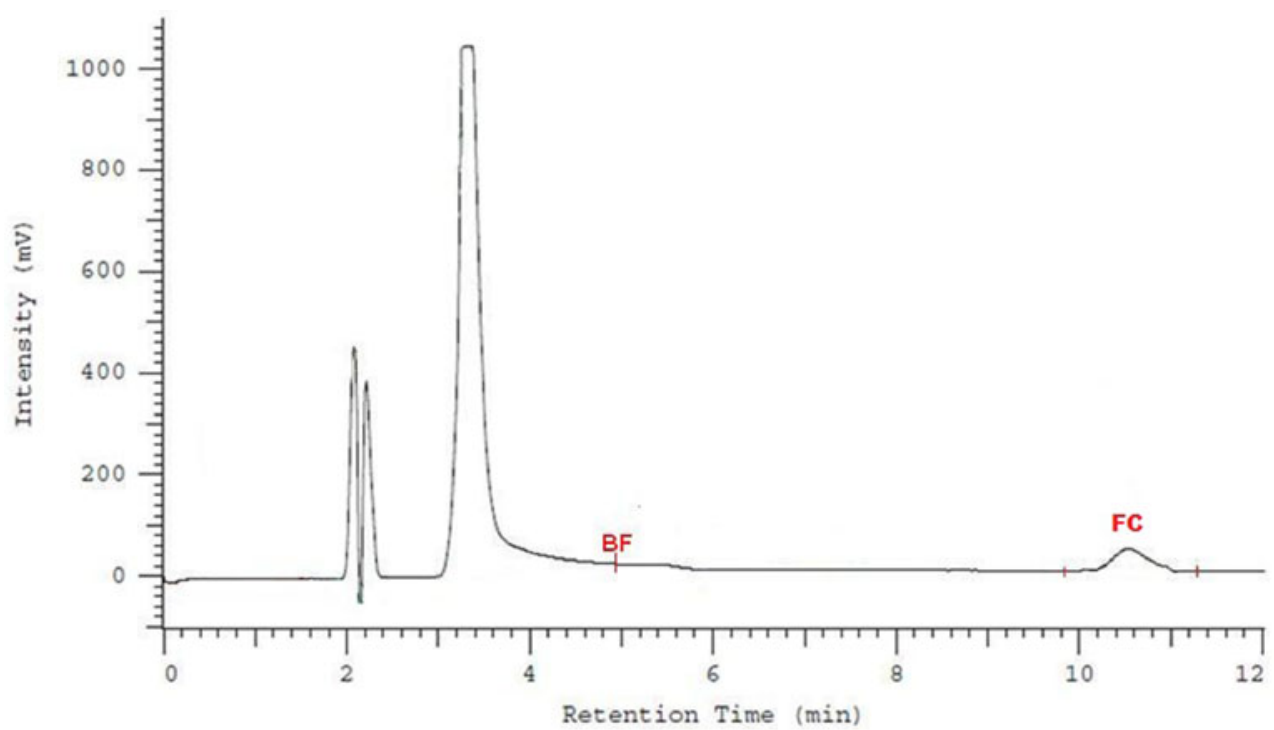




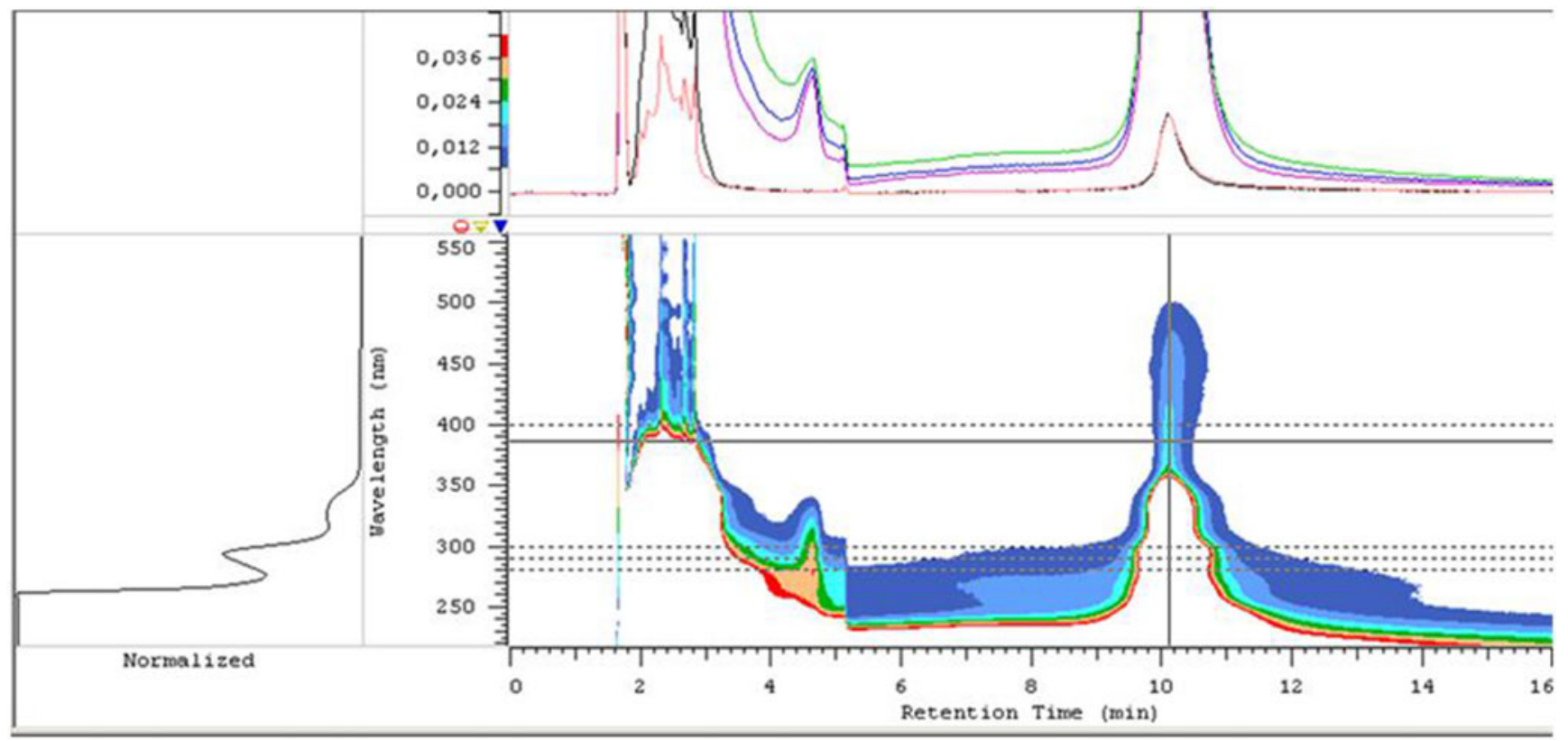

Fig. 3 HPLC/UV-VIS-DAD chromatogram of a sample of diesel fuel with the additive. Conditions as in Fig. 2

in the case of determination of lubricants and antioxidants $[9,11-13]$.

Consequently, it was necessary to isolate a characteristic fraction containing the additive components from diesel fuel using normal-phase adsorption HPLC, followed by quantitative determination by GC-FID or GC-MS with SIM.
The developed two-step analytical procedure involving isolation of a characteristic fraction of the additive by means of NP-HPLC allows separation of paraffins, cycloparaffins, alkenes and mono-, di- (including napthalene), and triaromatic compounds present in a sample of diesel oil (Fig. 1-backflush point at $12 \mathrm{~min}$, the additive peak collected

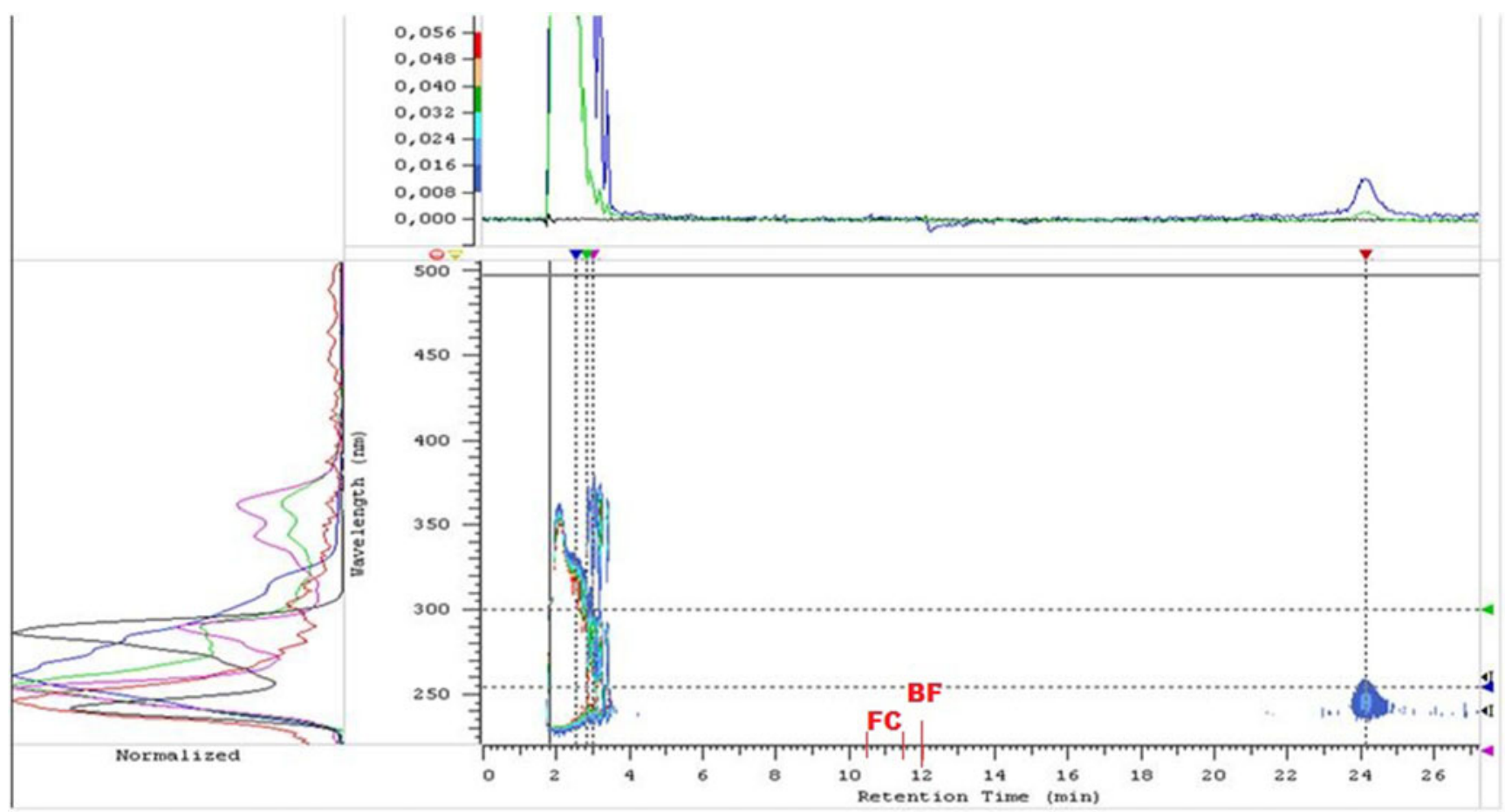

Fig. 4 HPLC/UV-VIS-DAD chromatogram of a sample of diesel fuel with the additive. Conditions as described in the section "Isolation of the additive fraction using NP-HPLC/UV-VIS-DAD/RID" of the
"Procedure"-50\% MTBE content in the mobile phase. Fraction collection range (FC): 10.5-11.5 min, $B F$ backflush point 


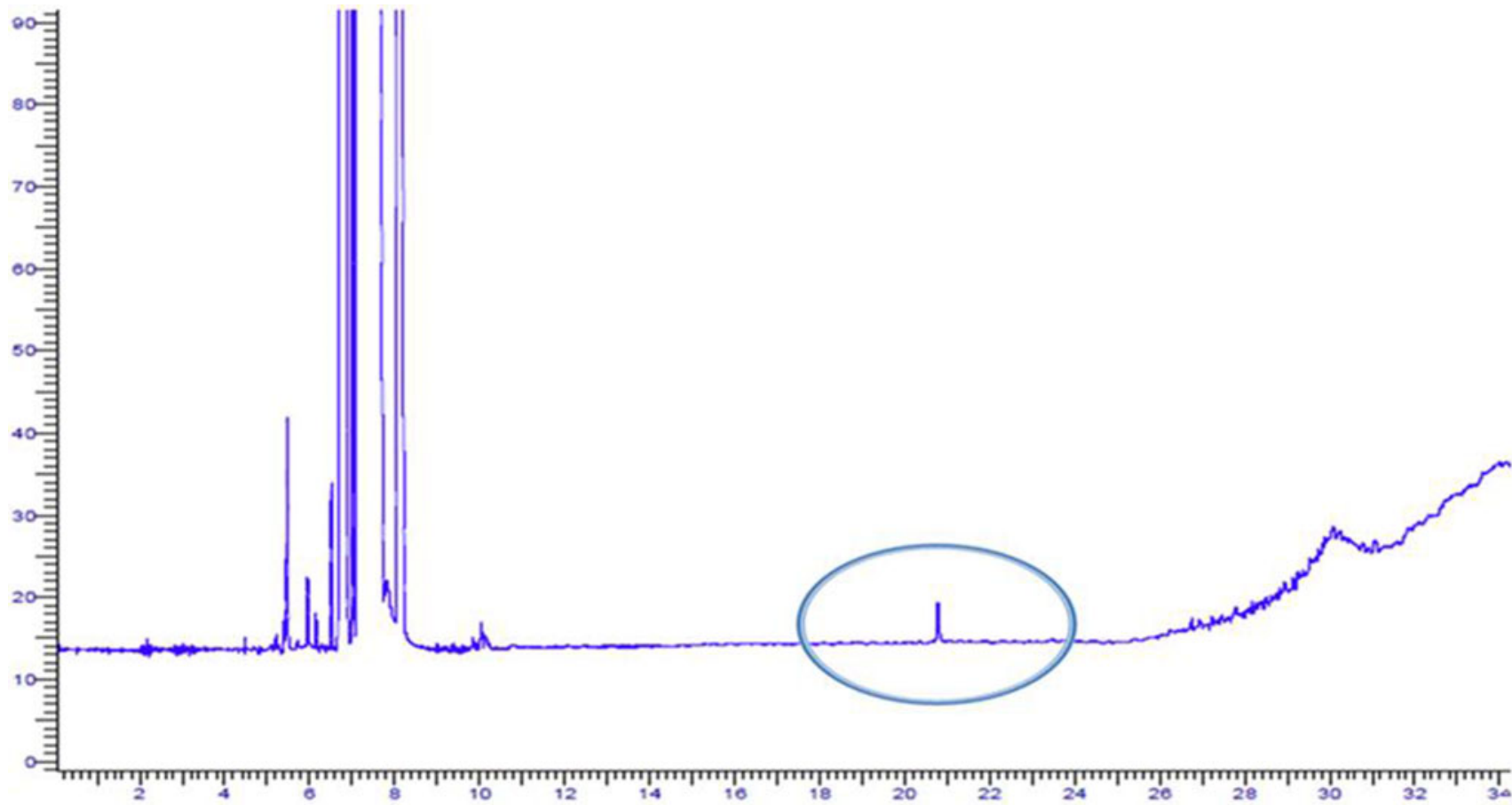

Fig. 5 GC-FID chromatogram from the two-step procedure NP-HPLC-EBF/GC-FID. The encircled peak corresponds to the 2-ethylhexanol in the additive fraction which was used for quantitative determinations

in backflush) from polar main components of cleaning additives, i.e., 2-ethylhexanol, which makes quantitative analysis by gas chromatography much easier. The use of pyrene as a marker determining eluent backflush point (EBF) results from the nature of group separation in normal HPLC, where pyrene is commonly employed as an indicator of the end of elution of tria-romatic compounds [14-16]. This enables removal of most of hydrocarbon components of the diesel fuel matrix from the collected fraction. The best composition of the mobile phase allowing satisfactory separation of individual groups of components was the mixture hexane/MTBE 8:1 $v / v$. Chromatograms corresponding to these conditions are shown in Figs. 2 and 3. Eluent backflush in the column (eluent backflush point: $5.0 \mathrm{~min}$ ) ensures complete elution of compounds introduced with the sample as one peak, whose start of elution corresponds to twice the backflush point (10 $\mathrm{min})$. Under such separation conditions, the additive fraction containing 2-ethylhexanol is collected during backflush. Diesel fuel can contain highly polar compounds (resins), whose strong UV absorption can be observed in Fig. 3 in the region of peak elution during backflush. In this case, the absence of eluent backflush would require step elution using a solvent with high elution strength to ensure their complete removal from the column. Such an elution would require column equilibration at the initial composition of the mobile phase prior to every analysis. The use of a UV/DAD detector allows control of this type of components in a sample. A disadvantage of this approach is peak broadening in the first detector.

An advantage of elution of the additive fraction during the initial direction of flow of the eluent is a considerably smaller peak width and, therefore, a higher concentration of the collected fraction, which results in a higher sensitivity of the procedure. However, in this case it is necessary to use backflush or step elution following collection of the desired

Table 1 Compilation of analytical characteristics of the developed procedures

\begin{tabular}{llll}
\hline Method & $\begin{array}{l}\text { LOD/LOQ } \\
{\left[\mathrm{ppm}_{(w / w)}\right]}\end{array}$ & $\begin{array}{l}\text { Linear } \\
\text { range }\end{array}$ & $\begin{array}{l}\text { Reproducibility coefficient of variation } \\
(\mathrm{CV})[\%]\end{array}$ \\
\hline $\begin{array}{l}\text { Single-step GC-FID, GC-MS, NP-HPLC/UV/ } \\
\text { DAD/RID }\end{array}$ & No quantitative analysis possible & & \\
Two-step NP-HPLC-EBF/ GC-FID & $12 / 24$ & $24-1400$ & 1.8 \\
Two-step NP-HPLC-EBF/ GC-MS (SIM) & $1.1 / 2.2$ & $2.2-970$ & 4.3 \\
Two-step NP-HPLC/GC-FID & $4.8 / 9.6$ & $9.6-1400$ & 1.5 \\
Two-step NP-HPLC/GC-MS (SIM) & $0.7 / 1.4$ & $1.4-970$ & 4.0 \\
\hline
\end{tabular}


fraction in order to ensure complete elution of all of the sample components. A chromatogram obtained under these conditions is shown in Fig. 4. The additive fraction which was used for GC quantitative analysis (but is unseen in this HPLC chromatogram due to the lack of UV absorption by 2 ethylhexanol) was collected from 10.5-11.5 min (time of fraction collection $1 \mathrm{~min}$ ). A more concentrated fraction is obtained in this case compared to fraction collection using backflush (time of fraction collection $1.7 \mathrm{~min}$ ). An improvement in sensitivity of the determination is only marginal, however, and can be of significance only in the analysis of trace amounts of additive, and primarily in the case of contamination of one type of diesel fuel with another, containing an additive discussed in this paper.

A GC-FID chromatogram obtained using the two-step NP-HPLC-EBF/GC-FID procedure is shown in Fig. 5. Despite fraction collection under backflush conditions, which lowers selectivity of isolation of the fraction of interest, capillary chromatography of the isolated fraction allows accurate and precise quantitative determinations.

A comparison of characteristics of quantitative determinations using one- and two-step procedures studied in this work is compiled in Table 1. The single-step procedures were found to be ineffective even with highly selective detection parameters due to very complex sample matrices. On the other hand, validation procedure demonstrated that the two-step procedures yield accurate and reproducible results. In all, four alternative two-step procedures for the determination of cleaning additives in diesel fuel were developed.

The use of mass spectrometry during final determination enables additional quality control of the results through the measurement of ratio of intensities of ions with 70 and 83 $\mathrm{m} / \mathrm{z}$. On this basis, it is possible to determine peak purity, which in case of fuels with complex matrices is an additional advantage of this procedure.

The procedures described in Introduction allow direct onestep determination of components of diesel fuel additives, if they have a characteristic mass spectrum, by using GC-MS in the SIM mode or by other selective detection methods. For the additives studied in this paper, such a possibility does not exist, which was demonstrated by the results obtained when using one-dimensional techniques. Only the use of orthogonal separation through the $\mathrm{LC} \times \mathrm{GC}$ coupling, resolution enabling quantitative analysis is achieved.

\section{Conclusions}

A procedure for the determination of additives in diesel oil has been developed. The use of normal-phase highperformance liquid chromatography in the first step, followed by gas chromatography for the final determination allows determination of additives containing aliphatic alcohols, commonly used as components of diesel fuel cleaning additives. Depending on the separation conditions used during normal-phase LC, a characteristic fuel fraction containing this type of additives is collected either during separation or during backflush.

The obtained results demonstrate the possibility of accurate determination of the additive in diesel fuel. A wide linear dynamic range enables determinations of the additive content in control of blending process at a level of several hundred parts per million and also at the trace level. The lowest detection limit $(0.7 \mathrm{ppm} \mathrm{w/w})$ is obtained when using NP-HPLC in the first step with fraction collection during normal flow, followed by gas chromatography-mass spectrometry in the SIM mode during final determination. This allows quality control of a fuel in case of the risk of contamination of a batch of fuel with a fuel of a different type, which can happen in a storage tank.

Open Access This article is distributed under the terms of the Creative Commons Attribution License which permits any use, distribution, and reproduction in any medium, provided the original author(s) and the source are credited.

\section{References}

1. Cook BR, Berlowitz PJ, Wittenbrink RJ (1998) Diesel additive for improving cetane, lubricity, and stability. US Patent 5814109

2. Ching Yeh LI, Schlosberg RH, Miller RC, Bateman JR, Caers RF (2002), US Patent 6447557 B1: Diesel fuel composition

3. Diesel Cetane Booster, Material Safety Data Sheet, Barryman Products Inc. 3800 E. Randol Mill Rd. Arlington, 2005

4. Ribeiro NM, Pinto AC, Quintella CM, da Rocha GO, Teixera LSG, Guarieiro LLN, do Carmo Rangel M, Veloso MCC, Reyende MJC, Serpa da Cruz RS, de Oliveira AM, Torreset EA, de Andrade JB (2007) The role of additives for diesel and diesel blended (ethanol or biodiesel) fuels: a review. Energy Fuel 21:2433-2445

5. http://www.momentive.com/products/showtechnicaldatasheet.aspx? id $=14334$

6. Grabowski W, Haubrichs R (1999) Diesel fuel and lubricating oil antifoams and methods of use. US Patent 6:001,140

7. Boczkaj G, Kamiński M, Przyjazny A (2010) Process control and investigation of oxidation kinetics of postoxidative effluents using gas chromatography with pulsed flame photometric detector (GC-PFPD). Ind Eng Chem Res 49:12654-12662

8. Boczkaj G, Przyjazny A, Kamiński M (2011) New procedure for the determination of distillation temperature distribution of highboiling petroleum products and fractions. Anal Bioanal Chem 399:3253-3260

9. Chopra A, Singh D, Kalsi WR (2009) Determination of fatty acid based lubricity improver in diesel by GC. Chromatographia 70:1143-1146 
10. Gorecki T, Martos P, Pawliszyn J (1998) Strategies for the analysis of polar solvents in liquid matrixes. Anal Chem 70:19-27

11. Rezende MJ, Perruso CR, de Azevedo DA, Pinto AC (2005) Characterization of lubricity improver additive in diesel by gas chromatography-mass spectrometry. J Chromatogr A 1063:211-5

12. Bernabei M, Bocchinfuso G, Carrozzo P, De Angelis C (2000) Determination of phenolic antioxidants in aviation jet fuel. $\mathrm{J}$ Chromatogr A 871:235-241

13. Bernabei M, Secli R, Bocchinfuso G (2000) Determination of additives in synthetic base oils for gas turbine engines. J Microcolumn Sep 12:585-592
14. EN 12916:2000, Petroleum products. Determination of aromatic hydrocarbons types in middle distillates. High performance liquid chromatography with refractive index detector.

15. Kartanowicz R, Przyjazny A, Kamiński M (2004) Application of high-performance liquid chromatography with ultraviolet diode array detection and refractive index detection to the determination of class composition and the analysis of gasoline. J Chromatogr A 1029:77-85

16. Gilgenast E, Boczkaj G, Przyjazny A, Kamiński M (2011) Sample preparation procedure for the determination of polycyclic aromatic hydrocarbons in petroleum vacuum residue and bitumen. Anal Bioanal Chem 401:1059-1069 